\title{
Percutaneous or surgical access for transfemoral transcatheter aortic valve implantation
}

\author{
Amit N. Vora, Sunil V. Rao \\ Duke University Medical Center, Duke Clinical Research Institute, Durham, NC, USA \\ Contributions: (I) Conception and design: All authors; (II) Administrative support: SV Rao; (III) Provision of study materials or patients: None; (IV) \\ Collection and assembly of data: All authors; (V) Data analysis and interpretation: All authors; (VI) Manuscript writing: All authors; (VII) Final \\ approval of manuscript: All authors. \\ Correspondence to: Sunil V. Rao, MD. Duke University Medical Center, Duke Clinical Research Institute, 508 Fulton St (111A), Durham, NC, USA. \\ Email: sunil.rao@duke.edu.
}

\begin{abstract}
Transcatheter aortic valve implantation is an important therapeutic option for patients with symptomatic, severe aortic stenosis at increased risk for open surgical aortic valve replacement. Although a number of alternative vascular access sites have been developed, transfemoral access is overwhelmingly the dominant access strategy for this procedure. Access was achieved in the initial clinical experience primarily via surgical cutdown, but more recently, there has been increasing use of a fully percutaneous approach. This is likely driven by improvements in technology, more experience with large bore vascular closure devices (VCDs), and pressures to reduce hospital length of stay. This review will describe temporal trends of percutaneous versus surgical transfemoral access, identify the advantages and disadvantages of each approach, and describe the best practices for achieving safe transfemoral percutaneous access.
\end{abstract}

Keywords: Aortic valve; TAVR; vascular access

Submitted Jul 04, 2018. Accepted for publication Sep 14, 2018.

doi: $10.21037 /$ jtd.2018.09.48

View this article at: http://dx.doi.org/10.21037/jtd.2018.09.48

Transcatheter aortic valve implantation (TAVI) has emerged as an important therapeutic option in the management of severe, symptomatic aortic valve stenosis, initially in patients at prohibitive risk for open surgical replacement but more recently in patients at high and intermediate risk for surgery. As the indications for TAVI have progressed to include more patients at the lower end of the risk spectrum, vascular access has emerged as an important criterion with which to evaluate not only procedural success but also overall safety and efficacy when compared with a surgical option (1). In the initial PARTNER A trial, access options were limited to transfemoral or transapical access, and $71 \%$ of patients underwent transfemoral access, with sheath sizes between 22-24 French, depending on the size of the implanted valve $(2,3)$. In a temporal trends analysis of the PARTNER continued access registry, surgical cutdown was employed in over two-thirds of initial patients but only one-third of patients enrolled later in the study (4). In the commercial use of the Medtronic CoreValve, surgical cutdown was used in only $20 \%$ of patients undergoing placement of the Evolut R platform (5). Since the initial PARTNER study, additional access options have been developed, including axillary, carotid, direct aortic (6), and transcaval (7). Nevertheless, femoral access continues to be the most important access point and is currently used in the vast majority of patients in the United States.

During the early clinical experience with TAVI, transfemoral access was achieved using a surgical cutdown approach. Since that time, however, there has been accumulating evidence of the safety and efficacy of a fully percutaneous approach. There are likely three main reasons that a fully percutaneous approach is being utilized more frequently. First, iterative improvements in the delivery platforms have substantially decreased the sheath size, down from 22-24 Fr to now 14 Fr for the commercially available valve platforms. Next, because hemostasis following a 
percutaneous procedure cannot be achieved by manual compression only, increasing experience with vascular closure devices (VCDs) has allowed more operators to be comfortable with closure of large arteriotomy sites. Finally, with increasing pressure to reduce overall procedural time and hospital length of stay, fully percutaneous access in appropriately selected patients may be an attractive option to make the procedure as minimally invasive as possible.

A number of studies have compared transfemoral surgical versus percutaneous access. An initial pilot study by Holper and colleagues randomized $n=30$ patients undergoing transcatheter aortic valve replacement (TAVR) using the initial SAPIEN platform (requiring 22-24 French delivery sheath) and found similar rates of Vascular Access Research Consortium-2 (VARC-2) major and minor complications (8), all of which required percutaneous or surgical intervention. In a retrospective analysis of $n=274$ patients undergoing TAVR by Nakamura and colleagues, 140 patients underwent a fully percutaneous procedure, with the remaining undergoing a surgical cutdown. In this analysis, rates of isolated stenosis or dissection were higher among patients undergoing percutaneous access $(7.1 \%$ vs. $0.7 \%, \mathrm{P}=0.007$ ) but wound infections requiring prolonged antimicrobial therapy or additional surgery were higher in the cutdown group ( $0.7 \%$ vs. $6.7 \%, \mathrm{P}=0.007)$, along with higher rates of blood transfusion $(25.7 \%$ vs. $43.3 \%$, $\mathrm{P}=0.002$ ) (9). Importantly, median hospital length of stay was also significantly shorter in the percutaneous group versus the surgical cutdown group ( 3 vs. 4 days, $\mathrm{P}=0.002$ ). In an analysis of the Spanish TAVI registry, a percutaneous approach was associated with higher minor vascular complications $[15 \%$ vs. $4.1 \%$, risk ratio (RR) $3.56,95 \%$ confidence interval (CI), 2.32-5.47] but lower rates of major bleeding (3\% vs. 6.6\%, RR 0.45, 95\% CI, 0.26-0.78) (10).

Because it can be extremely challenging to achieve hemostasis of large bore access with manual pressure only, one key aspect in the transition from surgical cutdown toward a fully percutaneous procedure has been more increasing experience and widespread use of VCDs. In the percutaneous coronary intervention (PCI) literature, studies have been somewhat conflicting but have not demonstrated a decreased rate of vascular access complications with the use of VCD compared with manual compression (11-14). Nevertheless, these devices are critical to ensuring the success of a fully percutaneous procedure. A recent metaanalysis of 17 studies including 7,889 vascular access sites for large bore vascular access in endovascular aneurysm repair (EVAR), thoracic endovascular aneurysm repair (TEVAR), and TAVR demonstrated that compared with surgical cutdown of the common femoral artery, VCD use was associated with fewer post-operative seromas [odds ratio (OR) 0.15 ; 95\% CI, 0.06-0.35], less wound dehiscence (OR 0.14; 95\% CI, 0.03-0.78), and fewer surgical site infections (OR 0.38 ; 95\% CI, 0.23-0.63), although postoperative pseudoaneurysms were more frequent in the VCD group (OR 3.83; 95\% CI, 1.55-9.44) (15). Unlike the other analyses noted above for TAVI, overall length of stay was not different between the two groups.

\section{Best practices for transfemoral percutaneous access}

\section{Before the procedure}

Prior to the TAVI procedure, the most important step in minimizing vascular access complications is meticulous pre-procedural planning. Almost all patients undergoing TAVI in the United States undergo computed tomographic angiography (CTA) to assess the vascular anatomy. Ensuring appropriate vascular anatomy is critical to ensuring a successful percutaneous approach. On the initial CTA, it is important to note the minimal luminal diameter of each vessel along with calcium burden, bifurcation height, and the presence of dissection or soft plaque. The instructions for use for the Medtronic Corevalve require a minimum diameter of $5.0 \mathrm{~mm}$ for the $14 \mathrm{Fr}$ delivery system and $5.5 \mathrm{~mm}$ for the $16 \mathrm{Fr}$ system, whereas the SAPIEN S3 platform requires a minimum of 5.5 and $6.0 \mathrm{~mm}$ for the $14 \mathrm{Fr}$ and the $16 \mathrm{Fr}$ E-sheaths, respectively.

\section{During the procedure}

It is important to use both fluoroscopic and ultrasound guidance to ensure the optimal percutaneous stick. Comparing the spatial relationship between the femoral head and the common femoral artery on the CTA will be helpful in identifying the appropriate fluoroscopic landmark for vascular access. Additionally, ultrasound guidance will allow for access at a site that is devoid of calcium or soft plaque while ensuring that the site remains above the common femoral artery. After the vessel is accessed, repeat fluoroscopic imaging can be helpful to ensure that the access site is not too high.

The Pre-close technique is most commonly used to prepare for percutaneous vascular closure following the 
procedure. After the vessel is accessed and the guidewire is advanced, two Proglide devices are deployed at orthogonal angles (i.e., the device is angled about 45 degrees facing and away from the operator) prior to the placement of the delivery sheath.

\section{Following valve deployment}

Once valve implantation is complete, procedural focus is directed toward vascular access closure and hemostasis. Over a stiff guidewire, the sheath is removed while both operators are simultaneously cinching the Per-close sutures to achieve hemostasis. As long as pulsatile flow is not observed, the guidewire is removed, the sutures are locked and cut, and then manual pressure is applied until hemostasis is achieved. If the sutures do not adequately seal the vessel, options to achieve hemostasis include deploying a third Per-close suture (if the guidewire is in place), obtaining wire access from the contralateral femoral artery and deploying a covered stent, or surgical cutdown for primary repair of the vessel.

\section{Conclusions}

Vascular access management is a critical piece of a successful TAVI procedure. Overwhelmingly, transfemoral access is the access site of choice, and while surgical cutdown was the preferred access strategy in early clinical experience, recent trends demonstrate increased use of a percutaneous approach. This trend is likely to continue due to additional device iterations and enhancements, increasing operator experience with VCD use as well as dedicated devices for large-bore closure, and added pressure reduce procedural and post-procedural hospitalization costs to make the procedure as minimally invasive as possible. Meticulous preprocedure planning, accurate percutaneous access utilizing both fluoroscopy and ultrasound, and careful deployment of the VCDs are critical steps to ensure a successful percutaneous procedure.

\section{Acknowledgements}

None.

\section{Footnote}

Conflicts of Interest: The authors have no conflicts of interest to declare.

\section{References}

1. Genereux P, Webb JG, Svensson LG, et al. Vascular complications after transcatheter aortic valve replacement: insights from the PARTNER (Placement of AoRTic TraNscathetER Valve) trial. J Am Coll Cardiol 2012;60:1043-52.

2. Leon MB, Smith CR, Mack M, et al. Transcatheter Aortic-Valve Implantation for Aortic Stenosis in Patients Who Cannot Undergo Surgery. N Engl J Med 2010;363:1597-607.

3. Smith CR, Leon MB, Mack MJ, et al. Transcatheter versus Surgical Aortic-Valve Replacement in High-Risk Patients. N Engl J Med 2011;364:2187-98.

4. Beohar N, Kirtane AJ, Blackstone E, et al. Trends in Complications and Outcomes of Patients Undergoing Transfemoral Transcatheter Aortic Valve Replacement: Experience From the PARTNER Continued Access Registry. JACC Cardiovasc Interv 2016;9:355-63.

5. Sorajja P, Kodali S, Reardon MJ, et al. Outcomes for the Commercial Use of Self-Expanding Prostheses in Transcatheter Aortic Valve Replacement: A Report From the STS/ACC TVT Registry. JACC Cardiovasc Interv 2017;10:2090-8.

6. Ramlawi B, Anaya-Ayala JE, Reardon MJ. Transcatheter aortic valve replacement (TAVR): access planning and strategies. Methodist Debakey Cardiovasc J 2012;8:22-5.

7. Greenbaum AB, O'Neill WW, Paone G, et al. Caval-aortic access to allow transcatheter aortic valve replacement in otherwise ineligible patients: initial human experience. J Am Coll Cardiol 2014;63:2795-804.

8. Holper EM, Kim RJ, Mack M, et al. Randomized trial of surgical cutdown versus percutaneous access in transfemoral TAVR. Catheter Cardiovasc Interv 2014;83:457-64.

9. Nakamura $M$, Chakravarty T, Jilaihawi H, et al. Complete percutaneous approach for arterial access in transfemoral transcatheter aortic valve replacement: a comparison with surgical cut-down and closure. Catheter Cardiovasc Interv 2014;84:293-300.

10. Hernandez-Enriquez M, Andrea R, Brugaletta S, et al. Puncture Versus Surgical Cutdown Complications of Transfemoral Aortic Valve Implantation (from the Spanish TAVI Registry). Am J Cardiol 2016;118:578-84.

11. Patel MR, Jneid H, Derdeyn CP, et al. Arteriotomy closure devices for cardiovascular procedures: a scientific statement from the American Heart Association. Circulation 2010;122:1882-93. 
12. Marso SP, Amin AP, House JA, et al. Association between use of bleeding avoidance strategies and risk of periprocedural bleeding among patients undergoing percutaneous coronary intervention. JAMA 2010;303:2156-64.

13. Nikolsky E, Mehran R, Halkin A, et al. Vascular complications associated with arteriotomy closure devices in patients undergoing percutaneous coronary procedures: a meta-analysis. J Am Coll Cardiol 2004;44:1200-9.

Cite this article as: Vora AV, Rao SV. Percutaneous or surgical access for transfemoral transcatheter aortic valve implantation. J Thorac Dis 2018;10(Suppl 30):S3595-S3598. doi: 10.21037/ jtd.2018.09.48
14. Vaitkus PT. A meta-analysis of percutaneous vascular closure devices after diagnostic catheterization and percutaneous coronary intervention. J Invasive Cardiol 2004;16:243-6.

15. Vierhout BP, Pol RA, El Moumni M, et al. Editor's Choice - Arteriotomy Closure Devices in EVAR, TEVAR, and TAVR: A Systematic Review and Meta-analysis of Randomised Clinical Trials and Cohort Studies. Eur J Vasc Endovasc Surg 2017;54:104-15. 